\title{
Libros electrónicos: Del papiro al bit. Las implicaciones comunicacionales de un medio permeado por la tinta electrónica
}

\author{
Carlos Alberto GaLvis OrTIZ \\ Universidad de Medellín (Colombia) \\ cagalvis@udem.edu.co \\ María Isabel ZAPATA CÁRDENAS \\ Universidad de Medellín (Colombia) \\ mizapata@udem.edu.co
}

\begin{abstract}
Resumen
Todo lo que llegue a ser digital lo será, incluyendo los libros. Con la consolidación de la Internet como gran sistema de comunicación global, los libros electrónicos representan hoy en día el siguiente nivel en la revolución digital. Más que la revolución del formato, es la revolución de los cambios significativos que se gestan en la producción digital de los contenidos. Centrarse en la adquisición y el desarrollo de la información, en la posibilidad de los multi formatos, como expresión de la narrativa transmedia, donde la convergencia y la multiplicidad se expresan en el escenario digital.
\end{abstract}

Palabras clave: sociedad TIC, E-books, Convergencia, Libros Electrónicos, Comunicación Digital, Dispositivos.

\section{E-books: From papyrus to bits. The implications of communication by means of electronic ink permeated}

\begin{abstract}
Everything becomes digital will be, including books. With the consolidation of the Internet as a great global communication system, electronic books today represent the next level in the digital revolution. More format that revolution is the revolution of the significant changes that are developing in the digital production of content. Focus on the acquisition and development of information, the possibility of multi formats such as expression of transmedia storytelling, where the convergence and multiplicity are expressed in the digital arena.
\end{abstract}

Key words: Digital Society, E-Books, Electronic Books, Convergence, Digital Communication, Device.

\section{Referencia normalizada:}

Galvis Ortiz, C. A. y Zapata Cárdenas, M. I. (2013) Libros electrónicos: Del papiro al bit. Las implicaciones comunicacionales de un medio permeado por la tinta electrónica. Historia y Comunicación Social. Vol. 18. № Especial Diciembre. Págs. 237-247.

Sumario: 1. Introducción. 1.1. Metodología. 1.2. Del papiro al bit: Cuando la columna migró al pixel. 1.3. Las hipermediaciones. 2. Conclusiones. 3. Bibliografía 


\section{Introducción}

Con el surgimiento del libro electrónico se está produciendo una brecha de enormes dimensiones, si la comparamos con la producida en su momento entre la oralidad y la escritura, o entre la escritura impresa respecto de la manual: entre la aparición de la imprenta de tipos móviles ${ }^{1}$ (Johannes Gutenberg, 1450), y la narración tribal, de generación en generación.

Con la llegada de los libros digitales, el espacio de la interactividad de alguna manera es el gran escenario de experiencia frente al proceso de lectura.

"De lo interactivo se deduce la capacidad de participación por parte del receptor, con la consiguiente modificación del mensaje original, que es reconstruido según los intereses e interpretaciones del último descodificador; algo que nos lleva a cuestionarnos la alteración de la idea de autoría. Nos referimos a un nuevo autor-creador que se caracteriza por su pluralidad" (Caldevilla Domínguez, 2011).

Alrededor del libro electrónico se crea una industria nueva de editores, libreros y una nueva definición de autor como creador individual. Lo importante es el valor del contenido, donde los nuevos y modernos soportes propician nuevas formas para crear contenidos antes imposibles.

"El libro electrónico reemplaza las ideas de secuencialidad y causalidad por las de una actividad integrada y continua. En realidad en lugar de hablar de productos podríamos hablar de procesos en los que la obra se mantiene en una apertura permanente" (Cordón García, 2011).

En este contexto de desarrollo, pero también de ensayo y error, conviven en la actualidad el libro físico, y el libro electrónico (e-book). En la historia de las sociedades modernas, el libro impreso era considerado como el elemento transmisor cultural por excelencia, pero con la revolución digital el libro tradicional ha sido de los primeros en vivir las consecuencias derivadas de los avances en el mundo virtual: el nuevo modelo de libro digital, libro electrónico, e-libro. Convivencia entre lo análogo y lo digital, entre el olor de la página y la tinta electrónica que alivia la lectura.

\subsection{Metodología}

Dado el vertiginoso desarrollo registrado a lo largo de los últimos años, la comunicación se ha convertido en un área de acción fundamental para la construcción de la sociedad y la democracia. El avance de las nuevas tecnologías de la información y la comunicación -TIC- corre entrelazado con el proceso de globalización, como efecto y causa a la vez, al tiempo que la información se afirma como el principal insumo de los procesos productivos de punta y la comunicación como el proceso sin el cual no es posible la comprensión de las nuevas interacciones que se dan en la red y en lo social. Los paradigmas tradicionales, propios de una sociedad de masas del siglo XX, quedaron atrás. Hoy, la comunicación se concibe no desde el modelo tradicional de

1 Museo Gutenberg en Alemania: http://www.gutenberg-museum.de/ 
Harold Laswell o del modelo matemático de Shannon y Weaver, sino desde múltiples modelos cuyo común denominador radica en establecer nuevas interacciones sociales entre los actores que intervienen en el acto comunicativo. La sociedad de la información y no la sociedad de masas se abre paso, generando la posibilidad de pensar en que ésta sea un tipo de sociedad de transición y que la nueva sociedad que emerja sea la sociedad de públicos².

La "virtualización de la realidad", donde el contexto supera a la virtualidad o mejor la virtualidad es, en el hoy de la comunicación, la realidad misma o como algunos de los expertos en la red la están llamando la "realidad aumentada", las tecnologías de la información y la comunicación están generando una nueva forma de entender la comunicación y sus mediaciones. Al decir de Slavoj Žižek, un experto en el tema de los nuevos medios en el ciberespacio, "no era que antes había una realidad real y hoy hay sólo una realidad virtual, sino que a través de la experiencia de la realidad virtual nosotros nos hemos dado cuenta de algún modo retroactivamente de que nunca hubo una realidad real. La realidad siempre fue virtual y nosotros ni siquiera éramos conscientes de ello"3.

Desde este contexto los dispositivos y soportes móviles, son los espacios digitales ideales para continuar con la expansión del conocimiento. En la actualidad, cualquier información puede accederse desde los diferentes formatos que el desarrollo tecnológico ha incorporado al mercado, siendo los libros digitales (e-books) formatos con gran proyección dentro de la comunicación móvil. Formatos que enfrentan importantes desafíos en el ámbito de los contenidos y las aplicaciones digitales.

Los libros digitales presentan particularidades propias de la convergencia de medios, especificidades que ubican la experiencia del e-lector (e-reader) mientras se disfruta del video, el sonido, la animación y el juego interactivo, al ritmo del paso de las páginas y lectura en tinta electrónica. El presente texto forma parte de los resultados investigación y es el análisis estadístico e interpretación del trabajo encomendado por el Grupo de Investigación en Comunicación, Organización y Política- COP- de la Facultad de Comunicación, al Centro de Opinión Pública, relacionado con la Categorización de encuestas sobre la investigación "La Palabra Escrita en Tinta Electrónica. Las Nuevas Posibilidades Comunicativas de los Libros Electrónicos", al público en general, fondos editoriales y editoriales universitarias.

En su elaboración se utilizaron herramientas estadísticas apropiadas para alcanzar un resultado confiable, con el fin de proporcionarle al Grupo COP de la Facultad de

2 A propósito de la sociedad de públicos versus la sociedad de la información, se recomienda leer el texto de Luis Horacio Botero Montoya, titulado "Teoría de públicos. Lo público y lo privado en la perspectiva de la comunicación”, publicado por el Sello Editorial de la Universidad de Medellín, tercera edición, en enero de 2011, donde al autor hace una apuesta por la sociedad de públicos, con el fin de lograr una mayor y mejor democracia.

3 Para mayor información, consultar la entrevista realizada por Ulrich Gutmair y Chris Flor en junio de 1998 a SLAVOJ ŽIŽEK, titulada "Histeria y Ciberespacio".http://www.heise.de/bin/tp/issue/download.cgi?artikelnr=2492\&rub_ordner=inhalt, página consultada el 14 de agosto de 2011 a las 17:17. 
Comunicación un estudio que pusiera a su disposición los mayores elementos de juicio, para el logro de los objetivos formulados.

\subsection{Del papiro al bit: Cuando la columna migró al pixel}

El libro desde su aparición se convirtió en un hilo conductor de la democratización del conocimiento. Un elemento clave en la masificación de la información, gracias al acceso público a los múltiples contenidos que en formatos impresos fueron distribuidos por el alemán Johannes Gutenberg y su máquina tipográfica hacia 1450. La gran contribución alemana no sólo estuvo en el perfeccionamiento del proceso técnico sino en la masificación (para esta época) de la producción editorial. El Códice Iluminado elaborado ejemplar por ejemplar dio el brinco a la producción de textos por volumen. Toda una revolución industrial para la época. Una vez más la tecnología permeaba un proceso. Con el libro electrónico estamos asistiendo a una revolución del mundo digital. Si la multimedia, la participación social, la convergencia de medios han sido procesos vitales en la conformación de la gran red Internet, el turno ahora es para el sector editorial con la llegada y el ofrecimiento en el mercado de la publicación electrónica de libros. Y para entender lo que sucede es importante saber qué es un libro electrónico, o un e-book.

Pedro Sanz Baratz, en el artículo Libros electrónicos, el nuevo concepto del libro, explica que los libros "e-books o libros electrónicos son textos electrónicos que contienen características de formato especiales, las cuales permiten su lectura mediante software especializado. Los libros electrónicos tienen el aspecto de una pantalla, una pantalla que imita al libro o un libro que imita a la pantalla" (Sanz Baratz, 2007). Sobre esta definición el autor Sanz explica la denominación se relaciona tanto con el nuevo dispositivo de lectura diseñado para leer libros, como con los contenidos y programas que pueden correrse en su interior. Y cobija tanto a dispositivos de bolsillo, tabletas y lectores electrónicos. El libro electrónico es una realidad reciente que está disponible actualmente y que permite recibir miles de documentos o seleccionar uno a uno varios documentos, en el formato y peso de un libro en papel. Para facilitar la lectura, las obras se pueden editar según las preferencias personales: tipo y tamaño de letra, etc.

A finales de la década del noventa, luego de vivirse múltiples ensayos y experimentaciones que marcarían el camino de gestación del libro electrónico, el National Institute for Standars and Technology (NIST) de los Estados Unidos de América formó el Comité Abierto de Normas sobre e-books (OEBSC) al cual pertenecen la Association of American Publisher (AAP), Microsoft, Random House, y diferentes casas productoras y comercializadoras de lectores de e-books, cuya iniciativa permitió estandarizar el libro como hoy se conoce en el mercado.

El investigador Fernando Pérez Arranz, de la Universidad de Alcalá, explica que las "herramientas que incorpora el aparato electrónico aumentan notablemente sus funciones y sus posibilidades al permitir integrar a la vez texto, sonido, imágenes, permite realizar búsquedas, unir textos, etc. Permiten incluso subrayar párrafos o 
hacer anotaciones al margen, con lo que permiten más funciones que permite un libro tradicional y además potenciadas y mejoradas" (Pérez Arranz, 2001).

Según datos arrojados por la Asociación Americana de Editores de Libros, (the Association of American Publishers AAP), el año 2011 fue decisivo en la irrupción y recepción de los libros digitales frente a los modelos físicos. "En septiembre de 2011, las ventas de libros digitales llegaron a duplicarse, consiguiendo unos ingresos totales de \$80,3 millones de dólares" (Mellado, 2011). Explica el informe de AAP, que durante los primeros nueve meses de 2011, las ventas de libros digitales aumentaron un 137,9\% según informes de las editoriales americanas, hasta los 727,7 millones de dólares. Por otro lado, la misma Asociación reportó que durante el 2010, el e-book representó ya "el 8,3\% de la facturación total del sector" (Corral, 2011).

En el caso colombiano, la Cámara Colombiana del Libro (http://www.camlibro. com.co) explica en su Balance de la Agencia Colombiana de ISBN-ISMN del año 2010, que los registros de publicaciones impresas y digitales en el país toman fuerza, pero que aún es incipiente y bajo el nivel de producción.

"En el primer semestre de 2011 prevalecieron las publicaciones impresas en papel, pues se registraron 5.763 títulos correspondientes al $89 \%$ de la totalidad de los registros. El 11\% restante son de publicaciones disponibles en medios electrónicos, 789 títulos en total, entre los cuales se destacan los e-books (con un aproximado de 400 publicaciones)" (González, 2010).

Frente al tema de la producción, el común denominador en el formato es el $\mathrm{PDF}^{4}$. El uso constante de este formato lo ha posicionado como uno de los más representativos al momento de estructurar y publicar un libro electrónico. Estadísticas de la Cámara Colombiana del Libro ubican durante el 2010 al PDF como el formato líder en la producción de e-books en Colombia. El informe arroja que durante ese año el país suramericano produjo un total de "1.428 libros digitales, de los cuales el mayor porcentaje fueron en formatos PDF, HTML, Exe, y otros sin especificar" (González, 2010. Op. cit.).

Otro es el panorama y la realidad de la industria del libro digital en países como Estados Unidos. De acuerdo al portal de noticias $C N N$ en Español México "los estadounidenses con título universitario y de entre 30 y 49 años son quienes recurren más a la nueva modalidad de lectura. La tendencia a leer más libros electrónicos en EU coincide con el aumento en la posesión de tabletas electrónicas o dispositivos similares, como el iPad, el Kindle o el Nook. En 2011, un 18\% de los estadounidenses tenía algún dispositivo que permitía leer libros electrónicos; en 2012, lo tiene 33\%" (CNN en Español, 2012).

Hacia el año 2004, España presentó al mundo, el trabajo de la Fundación de "Grammata", entidad destinada al diseño, publicación y comercialización de libros electrónicos. Sin embargo la idea de tener un gran espacio digital y virtual donde se

4 PDF: Formato gráfico desarrollado por Adobe que reproduce un documento en formato digital para su posterior transmisión electrónica. (Anónimo, Diccionario Informático)

5 Grammata: http://grammata.es/ 
albergara miles de textos, fue propuesta una década atrás por la Biblioteca Virtual Miguel de Cervantes. Los avances presentados por Grammata empezaron a verse con el primer e-reader producido totalmente en España: Papyre, dispositivo que accedía a la lectura de libros digitales, revistas electrónicas y varios de los documentos electrónicos existentes en la época. Según el estudio El sector del libro en España 2010-2012. Observatorio de la Lectura y el Libro, "el incremento en la edición en otros soportes viene determinado por un crecimiento exponencial de la producción de libros digitales - categoría "Archivos de Internet" - , que han evolucionado de 12.948 registros en 2010 a 18.538 en 2011 —un +43,2\%" (Ministerio de Educación, 2010).

Hacia el 2009, la tienda norteamericana Amazon, llegaba a los mercados mundiales con su modelo de lector: Kindle. "las estimaciones apuntan a que el Kindle Fire ${ }^{6}$ podría haber vendido 3, 4 ó 5 millones de unidades. (...)IDC afirma que en el cuarto trimestre del año 2011 se vendieron 4,7 millones de Kindle Fire, lo que haría que Amazon tuviera un 16,8\% del mercado. La firma analista IHS dio una cifra considerablemente más baja, 3,89 millones, lo que equivaldría a una cuota del 14\%" (TICbeat, 2013).

El 2010, fue un año clave en el desarrollo, entrada al mercado y demanda de los dispositivos y los contenidos para libros digitales, además de la aparición de Libranda $^{7}$, primera plataforma creada por varias de las editoriales de lengua española y catalana más importantes, como: Planeta, Santillana y Mondadori; destinada a la distribución de libros digitales. Pero aún con estos esfuerzos, mercados como el mexicano demuestran otra cosa. En un informe del periódico El Universal de México, el panorama de la lectura y consumo de esta nueva tecnología sigue estando rezagada en el mercado mexicano. La razón: "La producción de libros digitales en México, apenas alcanza los mil 709 títulos al año, reporta la Cámara Nacional de la Industria Editorial Mexicana (CANIEM), sin embargo, el panorama de los libros electrónicos en México comienza a tomar forma, su venta comienza a generar impacto tanto en librerías como en Internet" (Aguilar Sosa, 2012).

Por otro lado, Brasil participaría de este boom con una nueva plataforma que funcionaría de la misma manera que Libranda: DLD (Distribuidora de Libros Digitales). Un repositorio con más de 1000 títulos, puestos a la venta directamente a las librerías, no al público final. Argentina por su parte, presentaría la plataforma Bubok, distribuidora de libros digitales, de carácter colaborativa donde los usuarios interesados en tener sus propias creaciones pueden hacerlo, creando toda una comunidad de lectores que comparten y comentan libros.

Frente a la situación de cómo incentivar y asumir el reto de generar una cultura no sólo de consumo sino de producción del libro digital, entidades del sector editorial

6 Tableta de la casa de libros Amazon.com que permite aplicaciones, juegos, libros, web, e-mail, eBooks publicados desde el portal de libros norteamericano.

7 Libranda es una empresa de servicios de distribución de contenidos editoriales digitales (ebooks) en todo el mundo. 
presenta en sus sitios web colombianos de venta en línea cifras importantes para el sector de los libros electrónicos. Norma S.A (hoy Carvajal Educación S.A.S) publica en su sitio web 537 títulos en formato electrónico, Editorial Planeta Colombiana S.A con 331 publicaciones y Librería de la $\mathrm{U}$, uno de los más grandes proveedores en estos formatos, asciende a 16 mil títulos. Todas estas transformaciones muestran un panorama general de como América Latina pese a muchas de sus dificultades económicas se adapta a los nuevos mercados y reconoce las posibilidades positivas de las nuevas formas de lectura.

\subsection{Las hipermediaciones}

Mientras los medios tradicionales se caracterizan, en mayor o menor medida, por acotar en unas dimensiones bien definidas sus contenidos (espacial y temporalmente), los medios digitales pueden ofrecer una mayor cantidad de información, dada su capacidad de almacenamiento; la posibilidad de actualizar continuamente los datos disponibles y de proporcionar la información a los usuarios que la solicitan desde cualquier parte del mundo y en cualquier momento. Desde este ámbito, los medios digitales abren la posibilidad de nuevas formas de mediación e interacción que se enriquecen con condiciones de tiempo, acceso, conectividad, rapidez y usabilidad, y redimensionan la interacción misma entre los sujetos y que se denominan modernamente como "hipermediaciones".

Empero el concepto de mediación es mucho más antiguo, ya que va desde los trabajos de los filósofos griegos como Aristóteles en el organón, donde expone en la doctrina del silogismo el llamado término medio, pasando por Agustín de Ipona en su texto Confesiones, donde resalta el carácter del verdadero mediador entre Dios y los hombres, hasta Hegel que en su texto de "Enciclopedia de las ciencias filosóficas", construye el concepto de mediación desde una perspectiva dialéctica. (Grisales y González, 2010: 119).

Para Hegel, la mediación se constituye en trascendencia, en movimiento del sujeto en su camino para constituirse como ser. El "ser en el ente", es decir, la esencia del ser, tiene sentido en la oposición sujeto-objeto vista en su temporalidad. Desde la mediación, el ser trasciende en su forma abstracta y la representación consciente de su ser inaugura el camino de la racionalidad.

Merleau-Ponty en las postrimerías de la modernidad y desde su análisis fenomenológico, va a sustentar el sentido de la mediación como percepción, a través del lenguaje, en especial en los campos de la comunicación y la cultura. Empero, el mayor desarrollo de la mediación en comunicación se da en la década de 1970 con la obra del investigador español Manuel Martín Serrano "La mediación social", donde aborda particularmente la mediación de los medios masivos de comunicación (léase mejor información) y las formas de mediación cognitiva y mediación estructural.

Desde el punto de vista cognitivo, la mediación equivaldría al sistema de reglas y de operaciones aplicadas a cualquier conjunto de hechos, o de cosas pertenecientes a planos heterogéneos de la realidad, para introducir un orden. (...) el mediador, 
por medio de "lo que ocurre" trata de explicar el orden. El suceso sirve para ilustrar una forma de consenso que ofrece un significado estable para interpretar el mundo. El mediador se sirve del acontecer para reproducir el código (Martín Serrano, 1997: 49-53).

Manuel Martín Serrano construye los significados de las formas de mediación cognitiva y estructural, y va a decir que:

A) El conflicto entre el cambio del acontecer y la reproducción de las normas sociales reclama una mediación cognitiva. La mediación cognitiva está orientada a lograr que aquello que cambia tenga un lugar en la concepción del mundo de las audiencias, aunque para proporcionarle ese lugar sea preciso intentar la transformación de esa concepción del mundo.(...) un medio interesado en preservar incólume la norma que exige respeto a los Derechos Humanos explicará su transgresión (la concurrencia de una muerte por tortura) como un acontecer aberrante o inadmisible; en todo caso procurará salvaguardar la legitimidad de la norma y no la del suceso. En cambio, puede aprovecharse de este hecho para erosionar el respeto hacia los Derechos Humanos. Presentará la muerte del detenido como un accidente y dará por sentado, explícita o implícitamente, la inevitabilidad e incluso la necesidad de que en la práctica policial se recurra a la tortura.

B) El conflicto entre la apertura del médium al acontecer imprevisto, y su cerramiento en una forma comunicativa, pide una mediación estructural. La mediación estructural está destinada a conseguir que aquello que irrumpe sirva para realimentar las modalidades comunicativas de cada medio productor. Es una tarea agónica en la que el medio se ve obligado a recurrir, para perpetuar su forma expresiva, a aquello que cada día vulnera los marcos formales de la comunicación, es decir, el acontecer imprevisible (Martín Serrano. 1997: 140-141).

Posteriormente, Jesús Martín-Barbero profundiza en el trabajo sobre mediación desde una mirada ecléctica, donde incorpora el estudio de la comunicación desde la cultura, el lenguaje y las prácticas sociales. Martín-Barbero desplaza las mediaciones de los medios a la articulación entre prácticas comunicativas, movimientos culturales y matrices sociales y va a decir que todas las instituciones normativas (escuela, familia, medios masivos de comunicación) son mediadoras en los procesos sociales y culturales. Lo que permite comprender la relación entre sistema social y sistema de comunicación, es la mediación (mutua afectación y cumplimiento de tareas).

En 1987, Martín-Barbero publica el texto De los medios a las mediaciones: comunicación, cultura y hegemonía, donde fundamenta su tesis de la mediación en comunicación desde el lugar de la recepción, es decir, desde la apropiación de los contenidos de los medios. Lo importante es que el proceso de comunicación se comienza a mirar desde las mediaciones y no desde los medios. Para Martín Barbero, las mediaciones son “articulaciones entre prácticas de comunicación y movimientos sociales” (Martín Barbero, 2003). Define más concretamente las mediaciones como "los lugares de los que provienen las constricciones que delimitan y configuran la materialidad social y la expresividad cultural (...) A modo de hipótesis, se propo- 
nen tres lugares de mediación: la cotidianidad familiar, la temporalidad social y la competencia cultural.” (Martín Barbero, 2003).

El modelo clásico que enfatiza que en la comunicación hay un emisor que envía un mensaje, a través de un canal, a un receptor o perceptor que recibe y percibe el mensaje y le da un uso y que luego este último se convierte en un emisor, para repetir el ciclo y lograr una retroalimentación o respuesta, ha sido, sencillamente, superado por no sólo un modelo, sino múltiples modelos de percepción y de interacción en la red. Hoy desde los dispositivos móviles y sus herramientas, la respuesta no es unívoca y no se da en el mismo tiempo y en el mismo espacio, Incluso, el interlocutor no es uno, sino varios y puede estar en distintos lugares al mismo tiempo e interactuando con muchos que no conoce, pero que empezó a conocer, a través de un simple clic. Las palabras usuario, colaborador, usabilidad, accesibilidad, interactividad, participación, tejido social, interfaces, contenidos, interacción, mediación, libros y hasta la misma comunicación adquieren nuevos significados.

Los cambios son multicausales y hacen que la forma tradicional de definir la comunicación, también sufra cambios sustanciales. Por ejemplo, uno de los teóricos de la comunicación, Manuel Castells, señala que "la comunicación en red y sus dispositivos posibilita la integración de todos los mensajes en un modelo cognitivo común, mediante la conjunción de soportes en una misma plataforma" (Castells, 2000: 404).

Así los libros digitales o E-book se convierten en una herramienta para auscultar nuevas formas de mediación social, que aunque no es objeto de esta investigación, permite dejar un camino abierto para desarrollar futuras indagaciones para analizar cómo los nuevos dispositivos móviles están generando interacción y mediaciones sociales, culturales y tecnológicas.

\section{Conclusiones}

El libro es un elemento digital que le permite no sólo acceder al conocimiento sino vivir la experiencia del lenguaje transmedial y convergente, en aquellos proyectos que desde su gestación se forman en la plataforma digital. Nacen y habitan en la esfera hipertextual y convergente. De lo contrario, son aproximaciones en formatos y estilos que migran al mundo digital, pero cuya concepción se da en el mundo análogo. Situación que el estudio confirma al referirse al tipo de formato más utilizado para crear libros digitales.

Pensar en el libro digital, es un salto que no sólo involucra avances tecnológicos. Su gran efecto y riqueza está en la posibilidad de alinearlo con procesos de inclusión, estudio y trabajo ciudadano, donde la tecnología sea una herramienta de mediación al servicio de los cambios sociales y de las necesidades de grupos humanos, que ven en la tecnología no solo un instrumento de vanguardia, sino un elemento de transformación humana que les posibilita mayor conocimiento, interactividad, nuevos formatos, distribución de la información a menor costo y exploración de la herramienta en 
sí. Innovación en este sentido no es sólo por ser una herramienta o un elemento de último generación, va acuñada al sentido de darle al ciudadano nuevas plataformas para innovar, soluciones digitales para sus necesidades empresariales, educativas o de emprendimiento.

Es retornar al papel del libro como primer vehículo de conocimiento y comunicación puesto en sus manos, esta vez a través de las Tecnologías de la Información y el Conocimiento.

\section{Referencia Bibliográficas}

AGUILAR SOSA, Y. (5 de Noviembre de 2012). Periódico El Universal. Recuperado el 20 de Mayo de 2013, de Periódico El Universal: http://www.eluniversal. com.mx/cultura/70255.html

BARBERO, Jesús Martín. De los medios a las mediaciones. Convenio Andrés Bello. Bogotá. 2003

CALDEVILla DOMÍNGUEZ, D. (2011). La Aparición Del Libro Electrónico (E-Book) Y Su Repercusión En El Libro Tradicional. Recuperado el 20 de Mayo de 2013, de La Aparición Del Libro Electrónico (E-Book) Y Su Repercusión En El Libro Tradicional: http://perio.unlp.edu.ar/ojs/index.php/question/article/ view/953/1037

CASTELLS, Manuel. The Rise of the Network Society: The Information Age: Economy, Society and Culture, Second Edition, Vol. 1, Blackwell, Oxford. 2000

CNN en Español. (30 de Diciembre de 2012). Recuperado el 18 de Septiembre de 2013, de CNN en Español: http://mexico.cnn.com/tecnologia/2012/12/30/el-numero-de-usuarios-de-libros-electronicos-en-eu-aumento-en-2012

CORDÓN GARCÍA, J. A. (2011). Lecturas sobre las lectura. Salamanca: Universidad de Salamanca.

CORRAL, M. d. (4 de Junio de 2011). Periódico El País. Recuperado el 13 de Septiembre de 2012, de El 'e-book' en busca de su primavera: http://elpais.com/ diario/2011/06/04/babelia/1307146333 850215.html

GONZÁlEZ, P. A. (2010). Cámara Colombiana del Libro . Recuperado el 14 de Septiembre de 2012, de Cámara Colombiana del Libro : http://licitaciones.camlibro.com.co/boletin/Estadisticas\%202010/Estadisticas\%202010.pdf

GRISALES, LM. y GONZALEZ, EM. "De un modo de relacionar dos elementos contrarios a la mediación o acerca de la aproximación histórica al concepto de mediación". En Revista Anagramas Volumen 9 Número 17. Sello Editorial Universidad de Medellín. Pp. 117-130. 2010.

Ministerio de Educación, C. y. (2010). El sector del libro en España 2010-2012. Madrid, España: Observatorio de la Lectura y el Libro.

MinTic. (Diciembre de 2011). MinTic Vive Digital. Recuperado el 01 de Mayo de 2013, de MinTic Vive Digital: http://www.mintic.gov.co/index.php/vive-digital/ plan/ecosistema/usuarios 
MinTIC. (1 de Diciembre de 2011). MinTIC: Diagnóstico y Punto de Partida de Colombia. Recuperado el 20 de Abril de 2013, de MinTIC: Diagnóstico y Punto de Partida de Colombia: http://www.mintic.gov.co/index.php/vive-digital/plan/ diagnostico-punto-partida-colombia

PÉREZ ARRANZ, F. (2001). El uso cotidiano de los libros electrónicos. Boletín de la Asociación Andaluza de Bibliotecarios, 9-25.

SANZ BARATZ, P. (6 de Enero de 2007). E-LIS repository. Recuperado el 11 de Abril de 2013, de http://eprints.rclis.org/8751/1/Documento1.pdf

SERRANO SANTOYO, A., \& Martínez Martínez, E. (2003). La Brecha Digital Mitos y Realidades. Baja California: Departamento Editorial Universitaria de la Universidad Autónoma de Baja California.

TICbeat. (31 de Mayo de 2013). Recuperado el 31 de Mayo de 2013, de TICbeat: http://www.ticbeat.com/sim/cuantos-kindle-fire-han-vendido/

\section{Los autores}

María Isabel Zapata es profesora de tiempo completo de la Facultad de Comunicación de la Universidad de Medellín, Programa de Comunicación Gráfica Publicitaria donde imparte las asignaturas de Taller Gráfico Publicitario V y Énfasis I para la Web: Pensamiento Digital. Ejerce como Coordinadora de Posgrados para la Facultad de Comunicación. Cuenta con experiencia en el campo TIC, portales para internet, gestión de proyectos de Contenido para la Web. Es magister en Periodismo y Comunicación Digital, de la Universidad Carlos III de Madrid.

Carlos Alberto Galvis Ortiz es profesor de tiempo completo de la Facultad de Comunicación de la Universidad de Medellín. Coautor del libro "Comunicación pública: repensar la comunicación para la democracia". (Sello Editorial Universidad de Medellín, junio de 2009). Comunicador Social y Periodista de la Universidad Jorge Tadeo Lozano de Bogotá. Licenciado en Español y Literatura de la Universidad del Quindío. Magíster en Educación (énfasis en Democracia) de la Universidad Católica de Manizales. Doctorando en Relaciones Internacionales Iberoamericanas, de la Universidad Rey Juan Carlos de España. 